\title{
Separation of bulk and surface-losses in low-loss EELS measurements in STEM
}

\author{
K. A. Mkhoyan ${ }^{1}$, T. Babinec ${ }^{1,2}$, S. E. Maccagnano ${ }^{1}$, E. J. Kirkland ${ }^{1}$, J. Silcox ${ }^{1}$ \\ ${ }^{1}$ School of Applied and Engineering Physics, Cornell University, Ithaca, New York 14853. \\ ${ }^{2}$ Physics Department, University of Michigan, Ann Arbor, Michigan 48109.
}

The popularity of analytical scanning transmission electron microscopy (STEM) as an alternative tool for characterization of materials is based primarily on its ability to carry out measurements on a scale as small as its probe size, which now can reach $0.8 \AA$ [1]. The emerging capability of improved energy resolution, for example down to $200 \mathrm{meV}$ or better, raises the interesting possibility of increasing the visibility of finer electronic states in the low energy loss region with atomic scale resolution. If quantitative analysis of spectra in this area of the spectrum is needed, better analysis than has hitherto been carried out will be necessary. In particular, the collective components of the spectrum (bulk plasmon, surface plasmon and Cherenkov radiation losses) need careful characterization. For example, separation of the bulk and surface plasmon energy loss spectra is required in order to invert the bulk response $\operatorname{Im}\{-1 / \varepsilon\}$ to find the true dielectric response function.

STEMs use a convergent beam as a probe and angle-integrated EELS, and as a result new limitations in the analysis of some EELS data are introduced. The response of the specimen to a fast electron propagating through it is a function not only of energy lost due to inelastic interactions, but also of the momentum transferred [2]. The inability to sort EELS by transferred momentum, $h q$, in the STEM limits analysis accessible in CTEM [3].

Here we present a new approach for the separation of bulk and surface losses in low-loss EELS spectra measured in STEM based on the assumption that the surface losses are independent of thickness above a particular thickness while the bulk losses vary linearly with thickness. Analysis shows that two low-loss EELS data measured at different thicknesses are sufficient. The limitations of the method are also studied. After separation of bulk and surface losses (see Fig. 1 and 2) each of them were treated separately. In the example of silicon we were able to obtain critical parameters characterizing the bulk plasmon excitation such as $E_{P, 0}$, the damping parameter $\Delta E_{P}$ and the coefficient of dispersion relation $\gamma$. This was achieved with a new numerical curve fitting procedure based on Multidimensional Simplex routine [4], where all parameters are determined simultaneously. The results are in good agreement with values reported in literature. A by-product of the analysis is a quantitative explanation of the shape of the angle-integrated plasmon energy loss.

The possibility of separating surface-losses from bulk-losses allows quantitative study of not only the features of the surface-loss but also the effects due to a coating of the sample with oxide layer. On the basis of the analysis we determine that the specimen was covered with a thin $10 \AA \mathrm{SiO}_{2}$ layer which was the result of few minutes' exposure to the air. Comparisons of the dielectric functions retrieved from EELS data with and without including surface-losses (see Figs. 3(a) and (b)) indicate that even for samples as thick as $800 \AA$ the effects of surface excitations can be significant [5].

References

[1] P. E. Batson, O.L. Krivanek, N. Dellby, Nature 418, 617 (2002). 
[2] H. Raether, in Vol 38 of Springer Tracts in Modern Physics, edited by G. Hohler (SpringerVerlag, Berlin 1965).

[3] P.E. Batson and J. Silcox, Phys. Rev. B 27, 5224 (1983).

[4] W.H. Press, S.A. Teukolsky, W.T. Vetterling, B.P. Flannery, Numerical Recipes in $\mathrm{C}^{++}$, (Cambridge University Press, 2002).

[5] This work is supported primarily by the Nanoscale Science and Engineering Initiative of the NSF EEC-0117770 and NYSTAR C020071. The sample preparation facilities and STEM are supported by NSF through the Cornell Center of Materials Research DMR 9632275. The technical support of M. Thomas is also acknowledged.
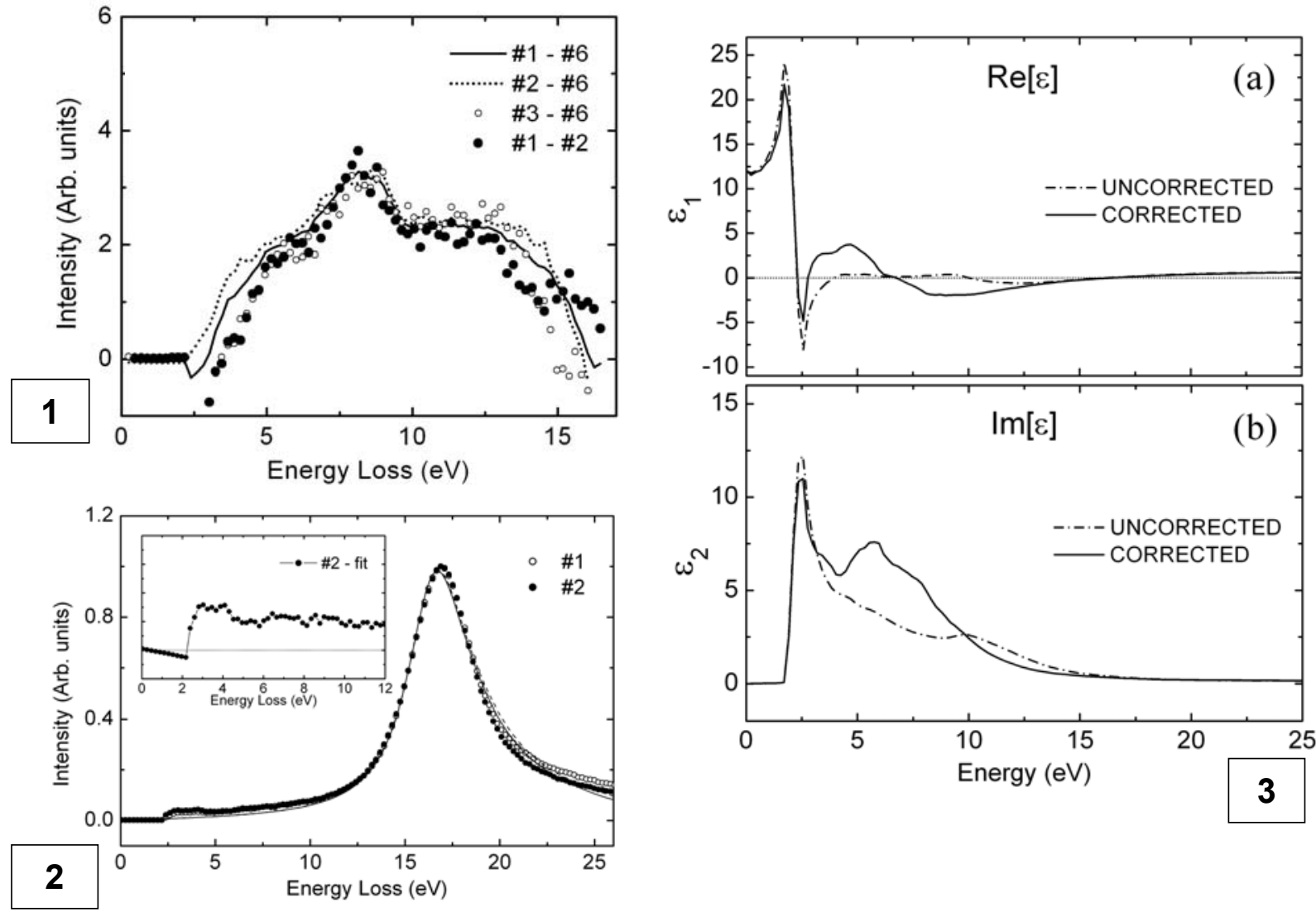

FIG. 1. Surface-loss spectra of silicon obtained from several pairs of normalized SSDs with different thicknesses.

FIG. 2. Bulk-losses in Si at thicknesses 320 and $420 \AA$, dominated by the plasmon-loss, with their fits. Dash line is fit to curve \#1 and solid line to curve \#2. The fitting procedure is based on numerical Multidimensional Simplex method. The inset shows the difference between spectrum $\# 2$ and its fit revealing small contributions from interband transitions and Cherenkov radiation.

FIG. 3. The real and imaginary parts of the dielectric function of Si deduced from SSD. The curves labeled 'corrected' correspond to results when surface-losses were removed. The specimen thickness is $420 \AA$ corresponding to SSD \#2 in Fig. 2. 